\title{
Reward Evokes Visual Perceptual Learning Following Reinforcement Learning Rules
}

\author{
Zhiyan Wang ${ }^{1}$, Dongho Kim ${ }^{1,3}$, Giorgia Pedroncelli ${ }^{4}$, Yuka Sasaki ${ }^{1}$ and Takeo Watanabe ${ }^{1,2}$ \\ ${ }^{1}$ Department of Cognitive, Linguistic and Psychological Sciences, \\ Brown University \\ 2takeo_watanabe@brown.edu \\ ${ }^{3}$ Asan Medical Center, 88 Olympic-ro 43-gil, Songpa-gu, Seoul 138-736, South Korea \\ ${ }^{4}$ Department of Human and Social Sciences, University of Bergamo, Piazzale S. Agostino 2, \\ Bergamo (BG) 24129, Italy.
}




\section{Abstract}

2

Visual perceptual learning (VPL) is defined as a long-term performance enhancement as a result of visual experiences. A number of studies have demonstrated that reward can evoke VPL. However, the mechanisms of how reward evoke VPL remain unknown. One possible hypothesis is that VPL is obtained through reward related reinforcement processing. If this hypothesis is true, learning can only occur when reward follows the stimulus presentation. Another interpretation is that VPL is acquired through an enhancement of alertness in association

with reward. If the alertness hypothesis is true, learning should occur when reward precedes the stimulus presentation. In our study, we tested the plausibility of the two hypotheses by manipulating the order of reward and stimulus presentation. In Experiment 1, we separated participants into two groups. During training, the 'Before' group received water reward 400ms prior to the onset of trained orientation stimulus while the 'After' group received water reward $400 \mathrm{~ms}$ subsequent to the onset of trained orientation stimulus. Both groups were trained using the Continuous Flash Suppression paradigm to render the stimulus imperceptible to the participants by the presentation of dynamic noise in the untrained eye. We found training only in the 'After' group indicating that reward may evoke learning through reinforcement-like processing. In Experiment 2, we excluded the possibility that alertness may not be sufficient to elicit learning when presented before stimulus. We presented beep sound prior to the onset of stimulus to increase alertness. Our finding demonstrated that alertness is sufficient enough to evoke learning. In conclusion, our study provided evidence that reward can evoke VPL through reinforcement process. 


\section{Introduction}

The role of reward in human behavior has been investigated comprehensively since

29 Pavlov's classical conditioning experiments(Rescorla \& Wagner, 1972; Wolfram Schultz, 2006;

30 W. Schultz, Dayan, \& Montague, 1997). How reward promotes learning has been a central

31 question. One of the most dominant models indicate that conditioning occurs when a stimulus is

32 predictive of reward (Rescorla \& Wagner, 1972). Schultz and his colleagues have found that

33 dopamine neurons in the substantia nigra were activated when rewards occurred at unpredicted

34 times and were depressed when rewards were omitted at the predicted times (Wolfram Schultz,

35 2002, 2007). They developed the theory in which conditioning is driven by the prediction error

36 between the reward expected and received.

Several studies have shown that reward also plays a critical role in promoting visual

perceptual learning (VPL) (Law \& Gold, 2008, 2009; Xue, Zhou, \& Li, 2015), which is defined

as a long-term performance improvement as a result of visual experiences (Dosher \& Lu, 2017;

Gilbert \& Li; Levi, 2012; Li, 2016; Li, Piëch, \& Gilbert, 2004; Sagi, 2011; Seitz \& Dinse, 2007;

41 Seitz, Kim, \& Watanabe, 2009; Watanabe \& Sasaki, 2015)

Seitz and his colleagues (Seitz et al., 2009) presented a sequence of two orientations in

43 random order which were made invisible by the continuous flash suppression paradigm

44 (Tsuchiya \& Koch, 2004) One orientation was paired with reward. The other orientation was not

45 paired with reward. All of the orientation stimuli were consistently presented in one eye for each

46 subject. The results showed that performance was enhanced only with the orientation paired with

47 reward and no transfer was found to the untrained eye. These results suggest that reward plays a

48 significant role in VPL, which is involved in early stages of visual processing. 
One possible interpretation of these results is that VPL is enhanced by reinforcement

50 processing. It has been found that learning of visual features is contingent on reward

51 probabilities, which is another necessity for reinforcement to occur (Kim, Seitz, \& Watanabe,

52 2015). Arsenault et al (Arsenault, Nelissen, Jarraya, \& Vanduffel, 2013) has also reported a

53 reward modulated decrease in BOLD signal in primate early visual cortex. Law \& Gold found

54 that response changes in the lateral intraparietal cortex (LIP) of monkeys fits well with VPL

55 driven by prediction errors (Law \& Gold, 2009). These and other studies are in accord with the

56 reinforcement-driven VPL hypothesis.

57 However, another possible interpretation is that reward increases alertness, which

58 enhances internal signals of a presented stimulus and leads to VPL. It has been suggested that

59 alertness can gate the processing of high priority information (Posner \& Petersen). Reward

60 increases the release of norepinephrine which in turn increases alertness, and therefore facilitates

61 visual processing (Aston-Jones \& Cohen, 2005; Dinse, Ragert, Pleger, Schwenkreis, \&

62 Tegenthoff, 2003). Specifically, stimulus paired with reward can evoke an increase in local field

63 potentials driven by enhanced attention(Frankó, Seitz, \& Vogels, 2010). Increased alertness

64 measured as dilated pupil diameters have facilitated visual contrast perception(Kim, Lokey, \&

65 Ling, 2017). It is noticeable that the above-mentioned Seitz study(Seitz et al., 2009), the last

$66100 \mathrm{msec}$ of an orientation presentation $(500 \mathrm{msec})$ was overlapped with reward delivery. This

67 further increases the possibility that reward enhanced an alertness level which directly enhanced

68 stimulus signals.

69 Although there have been proposals for both the reinforcement and the alertness

70 hypotheses in association with the mechanisms of reward, there is a lack of evidence that

71 indicate which of the two hypotheses is true. In this study, we examined the underlying 
72 mechanism of how reward evokes learning in lower-level visual feature processing with the task-

73 irrelevant learning paradigm. If the reinforcement hypothesis stands, for a stimulus to be learned,

74 the stimulus needs to be presented before reward so that the stimulus is predictive of reward. On

75 the other hand, if the alertness hypothesis stands, learning should occur when reward precedes

76 the stimulus. To address the question as to which hypothesis is true, we conducted an experiment

77 in which, a stimulus was presented before or after reward. We found that VPL occurred when a

78 stimulus was presented before reward, but VPL was not observed when it preceded reward. We

79 further found that VPL was not observed with the eye which is different from the eye in which

80 stimuli were presented during training, even when a stimulus was presented prior to reward.

81 These results rule out the alertness hypothesis and suggest that VPL occurs through interactions

82 between early visual areas and reinforcement processing that originates outside the visual system.

84 Results

Experiment 1

We conducted the first experiment using a typical perceptual learning procedure which

87 consists of 12 training sessions and the pre- and post- test sessions before and after the training,

88 see Figure 1 and Method. During the test sessions, orientation discrimination tasks were

89 performed to measure participants' sensitivity. During the training sessions, participants were

90 randomly separated into two groups which differed in the order of the presentations of reward

91 and stimuli. We presented a sequence of two oriented gratings only in the trained eye in both

92 groups. The Reward Before Stimulus ('Before') Group received water reward 400 ms before the

93 onset of the trained orientation presentation. The Reward After Stimulus ('After') Group

94 received water reward $400 \mathrm{~ms}$ after the onset of the trained orientation presentation. No reward 
95 was given accompanying the untrained orientation presentation. Moreover, we used the

96 Continuous Flash Suppression paradigm (Tsuchiya \& Koch, 2004) in which a continuous

97 sequence of high-contrast, contour-rich random patterns were presented to the untrained eye to

98 render the less salient stimulus in the trained eye imperceptible. The Continuous Flash

99 Suppression paradigm has been demonstrated as a robust paradigm that can cause invisibility of

100 the stimulus in the suppressed eye. The suppression effect can be reliably achieved from the

101 onset of the stimulus to avoid potential breakthroughs. Moreover, the effects of CFS is less

102 susceptible to the eye movement of the participants (Yang, Brascamp, Kang, \& Blake, 2014).

103 After the posttest, we conducted an awareness test and asked participants to indicate if they see

104 an orientated stimulus pattern and the orientation of it by pressing the corresponding button. The

105 stimulus was presented using the same paradigm as in training.

106

107

108

109

\section{0ms}
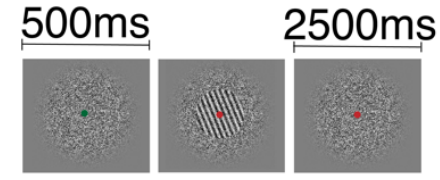

Noise Orientation Response

Time

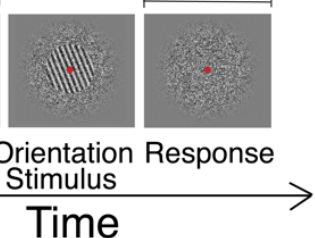

(A)

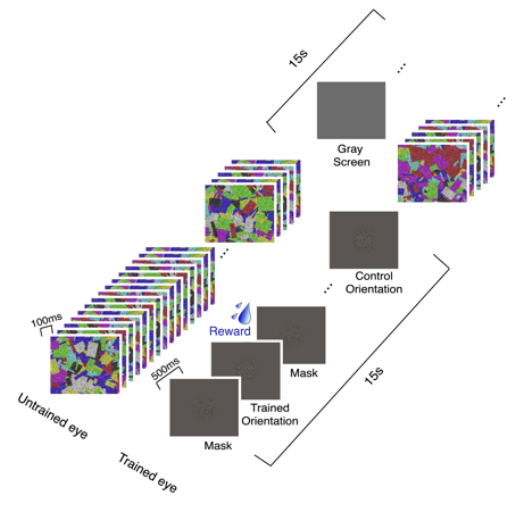

117

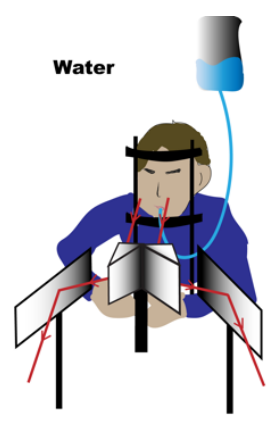

(B)

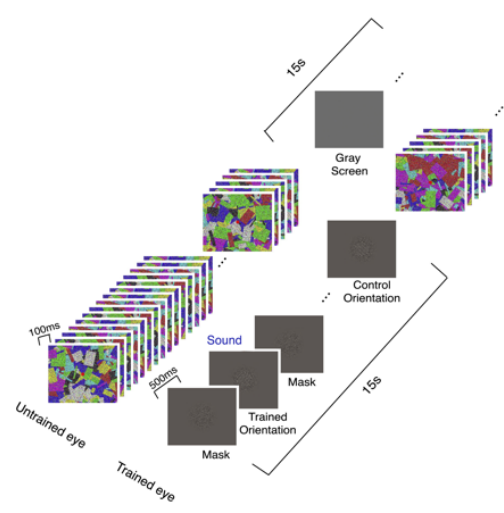

(D) 
Performance for the orientation discrimination task was characterized by the percentage

119 of correct responses under different S/Ns during pre- and post-test. The percent correct for two

120 orientations in the two eyes were shown in separate panels of Figure 2 for two groups 'Before'

121 and 'After' respectively. We calculated the improvement in performance as (Pretest-Posttest)/

122 Pretest. A three-way mixed model ANOVA was performed for improvement with 'Orientation',

123 'S/N ratio' as the within-group effect and 'Group' as the between-group effect. We found a

124 significant significant interaction of 'Group' $\mathrm{x}$ 'Orientations', $F(3,48)=3.46, p<0.05$. To rule

125 out the fact that, the improvement was a result of difference in pre-test performance, we

126 performed a separate ANOVA for the pretest performance with 'Group' as the between-group

127 effect and 'Orientation', 'S/N' ratio as the within group effect. We didn't find a significant effect

128 of the 'Group', $F(1,16)=0.458, p>.05$ or 'Orientation' $F(3,48)=2.64, p>.05$, or the

129 interaction between them, $F(3,48)=0.513, p>.05$. We only found a significant effect of ' $\mathrm{S} / \mathrm{N}$

130 ratio' in pretest performance, $F(6,96)=0.513, p<.001$. we performed separate ANOVA tests for

131 the 'After' group and the 'Before' group with 'Orientation $\mathrm{X} \mathrm{S} / \mathrm{N}$ ratio' as the main effect, we

132 didn't find a significant difference in the pretest performance for different Orientations, $F(3,24)$

$133=2.353, p>.05$ for the 'After' group and $F(3,24)=0.932, p>.05$ for the 'Before' group.

134 We performed repeated measures ANOVA in the 'After' group and the 'Before' group

135 separately with 'S/N ratio' and 'Orientation' as the within-subject factors. In the 'After' group,

136 we found a marginal significant effect of 'Orientation', $F(3,24)=2.404, p<.01$. There was no

137 significant effect in the 'Before' group.

138 In the 'After' group, post-hoc t tests showed that there was an improvement significantly

139 different from 0 in the trained eye and in the trained orientation specifically. At the $0.07 \mathrm{~S} / \mathrm{N}$

140 level, there was a significant improvement in percent correct after training with $t(8)=2.485$, 
$141 p<.05$. There was no significant improvement in the control orientations and in the untrained

142 eyes, all $p \mathrm{~s}>.05$. In the 'Before' group, we found a slight $10 \%$ improvement in the trained

143 orientation in the trained eye and the control orientation in the untrained eye only at the $0.2 \mathrm{~S} / \mathrm{N}$

144 level. No significant improvement was observed in the other conditions. As the stimuli at $0.2 \mathrm{~S} / \mathrm{N}$

145 level should be clear and conspicuous to the participants, the improvement in the $0.2 \mathrm{~S} / \mathrm{N}$ level

146 could be a result of improvement in performing the task itself.

147 In both groups during the awareness tests, participants rarely pressed button and reported

148 that they didn't see any oriented pattern. In the participants who responded, the accuracy was

$149 \quad 0.95 \% \pm 2 \%$ for the 'After' group and $2.78 \% \pm 8.33 \%$ for the 'Before' group.

150 To rule out the possibility that the improvement was a result of bias in response, we

151 analyzed the d' for both groups in the trained eye and the untrained eye. The d' for both eyes

152 were shown in Figure 3 for two groups 'Before' and 'After' respectively. Three-Way mixed

153 model ANOVA was performed with 'Group' as the between-group effect and 'Eye', 'S/N ratio'

154 as the within group effects. We found significant 'Group' main effect, $F(1,112)=10.626, p<.01$.

155 We also found significant 'Eye' $\mathrm{X}$ 'Group' interaction, $F(1,112)=5.179, p<.05$. We also found a

156 significant interaction of 'Eye' $\mathrm{X}$ 'S/N ratio', $F(6,48)=3.345, p<.01$. Post-hoc analysis

157 indicated that there is a significant improvement in trained eye at $0.1 \mathrm{~S} / \mathrm{N}$ ratio level, $t(7)=3.074$,

$158 p<.05$ and the $0.16 \mathrm{~S} / \mathrm{N}$ ratio level, $t(7)=2.39, p<.05$. 
(A) 'After' Group
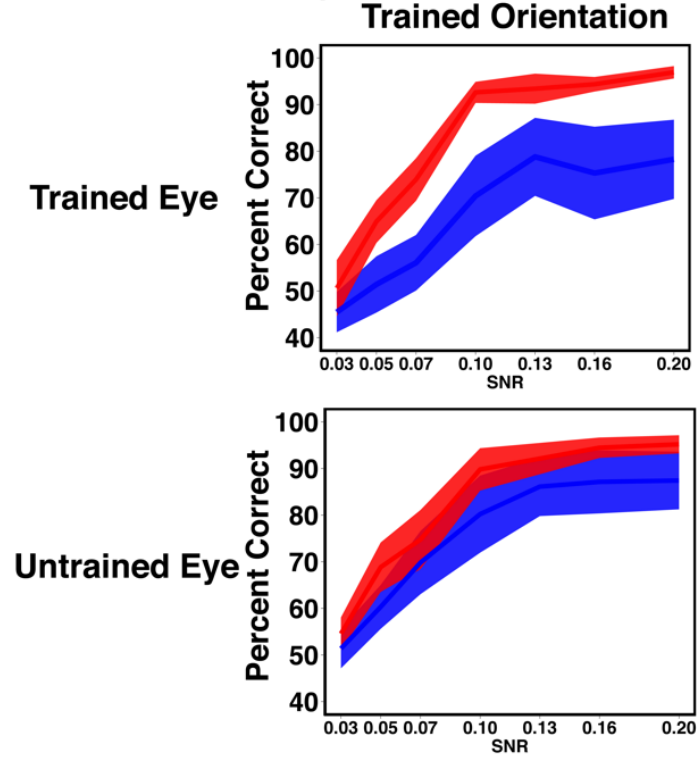

(A) 'Before' Group
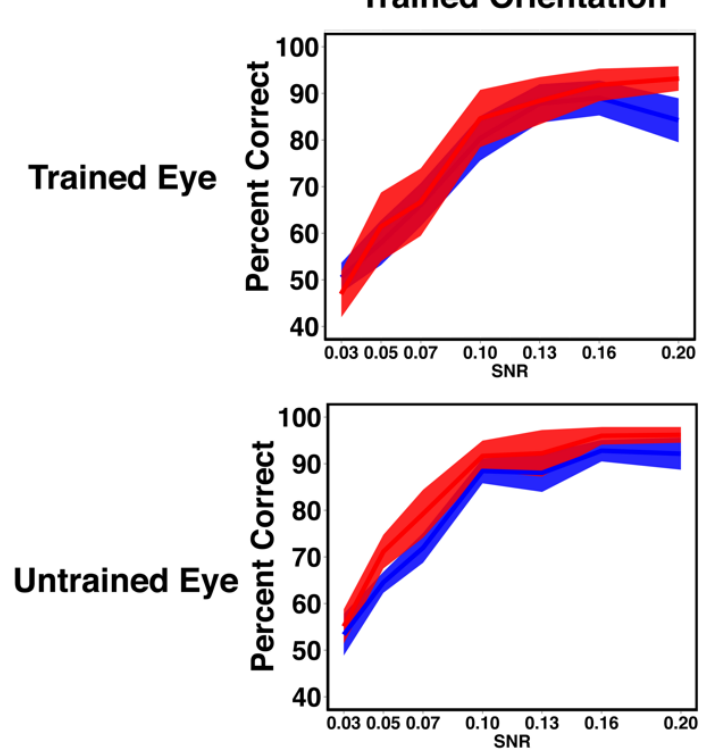
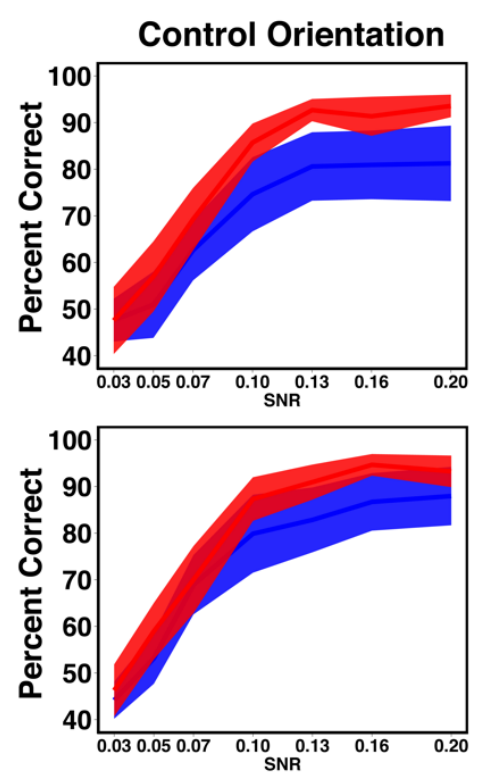

Pretest

Posttest
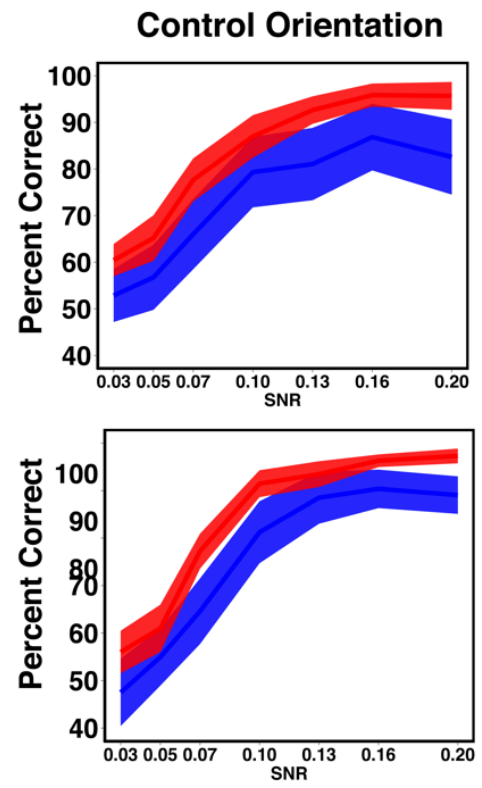

Pretest

Posttest

Figure 2. Result of Experiment 1. (A) Result for 'After' Group. Percent corret for Pretest and Post test measured for trained orienation and control orientation in the trained eye and untrained eye. Improvement was only found in the trained orientation in the trained eye. (B) Result for 'Before' group. Percent corret for Pretest and Posttest measured for trained orientation and control orientation in the trained and untrained eye. No significant improvement was found. 


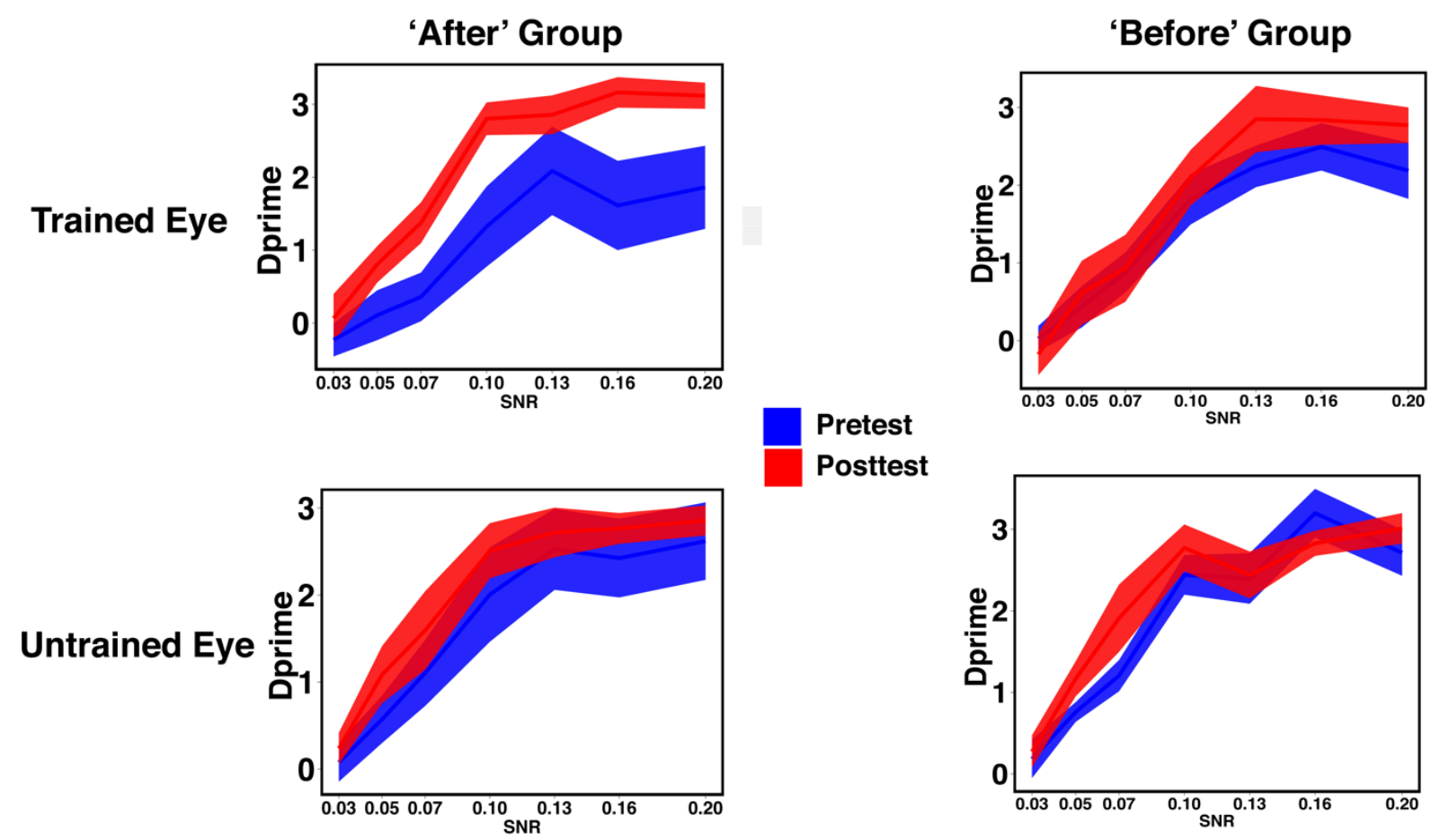

Figure 3. d' for Experiment 1 in two groups obtained with the trained eye and the untrained eye. There is a significant main effect of 'Group', $F(1,112)=10.626, p<.01$. and a significiant 'Group' $x$ 'Eye' interaction, $F(1,112)=$ $5.179, p<.05$. Post-hoc analysis showed significant impovement in d' in the traned eye of the 'After' group.

\section{Experiment 2}

There were at least two possible reasons why VPL of a visual stimulus did not occur

162 when reward preceded the stimulus in Experiment 1. The first is that VPL is driven by

163 reinforcement processing but not alertness enhancement. The second is that like reward,

164 somehow alertness needs to be enhanced after a stimulus for VPL. To test which possibility is

165 more likely, we conducted Experiment 2 in which we presented a beep, instead of reward, to

166 enhance alertness before stimulus presentation with the otherwise identical methods to those for

167 The Reward Before Stimulus ('Before') Group in Experiment 1.

As in Experiment 1, performance during pre- and post-test was characterized as

169 percentage of correct responses for orientation discrimination task under different $\mathrm{S} / \mathrm{N}$ ratios.

170 Percent correct for two orientations and two eyes were shown in separate panels in Figure 3. A 
171 two-way repeated measures ANOVA was performed with 'Orientation', 'S/N ratio' as the

172 within-group effects. We found a significant main effect of ' $\mathrm{S} / \mathrm{N}$ ratio', $F(6,42)=3.18, p<0.05$.

173 The main effect of 'Orientation', $F(3,21)=0.601, p>0.05$ and the interaction was not significant,

$174 F(18,126)=0.821, p>0.05$. Post-hoc analysis showed a significant improvement in percent correct

175 for the orientation that was paired with beep in the trained eye at the $0.1 \mathrm{~S} / \mathrm{N}$ level, $t(7)=3.002$,

$176 p<.05$. There is also a significant improvement in percent correct for the orientation that was

177 paired with beep in the untrained eye at the 0.1 and $0.13 \mathrm{~S} / \mathrm{N}$ level, $t(7)=2.88, p<.05$ and $t(7)=$

$1782.38, p<.05$ respectively. A significant improvement of the control orientation in the trained eye

179 was also observed at the $0.05 \mathrm{~S} / \mathrm{N}$ level, $t(7)=2.54, p<.05$.

As in Experiment 1, d's for the trained eye and the untrained eye were also analyzed and

181 shown in Figure 5. Two-way repeated measures ANOVA with 'Eye' and 'S/N' ratio as the

182 within-subject effects was performed. We found a significant main effect of the ' $\mathrm{S} / \mathrm{N}$ ' ratio,

$183 F(6,42)=3.012, p<.05$. Post-hoc analysis showed a significant improvement of the d' in the

184 trained eye at $0.1 \mathrm{~S} / \mathrm{N}$ ratio level, $t(7)=2.54, p<.05$ and significant improvement of the $\mathrm{d}^{\prime}$ in the

185 untrained eye at $0.1 \mathrm{~S} / \mathrm{N}$ ratio level $t(7)=2.73, p<.05$ and $0.13 \mathrm{~S} / \mathrm{N}$ ratio level, $t(7)=4.03, p<.01$.

186 Awareness tests were also conducted as in Experiment 1. The accuracy for participants

187 who responded during the awareness tests were $0.067 \pm 0.14$.

The results indicate that unlike reward alertness enhancement leads to VPL of a stimulus

189 even if alertness is enhanced before the stimulus presentation. This rules out the possibility that

190 alertness enhancement leads to VPL of a stimulus only when it occurs after the stimulus

191 presentation. Therefore, we conclude that VPL is driven by reinforcement processing. 

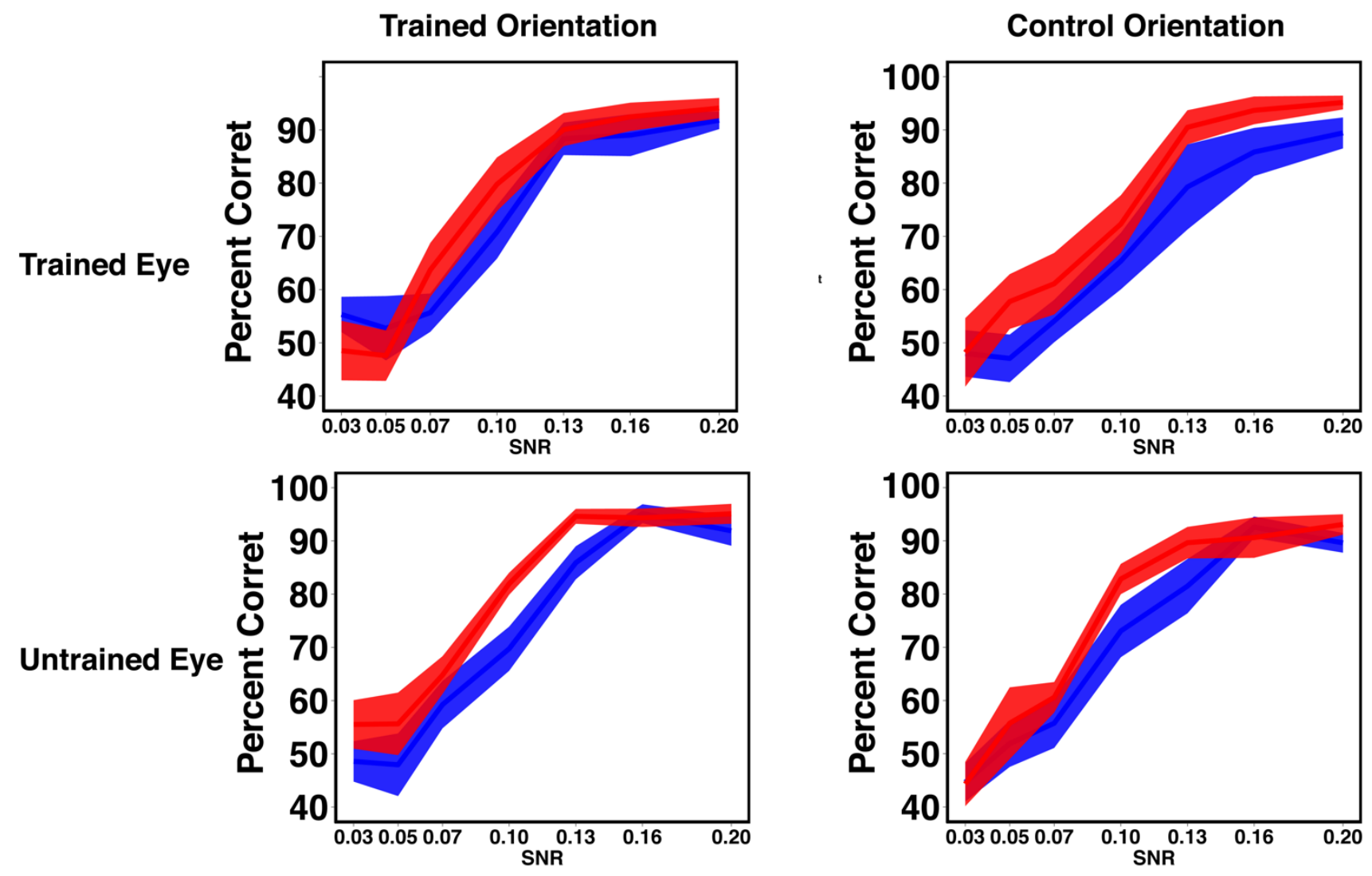

Figure 4. Result of Experiment 2. Percent correct for Pretest and Posttest measured for trained orienation and control orientation in the trained eye and untrained eye.
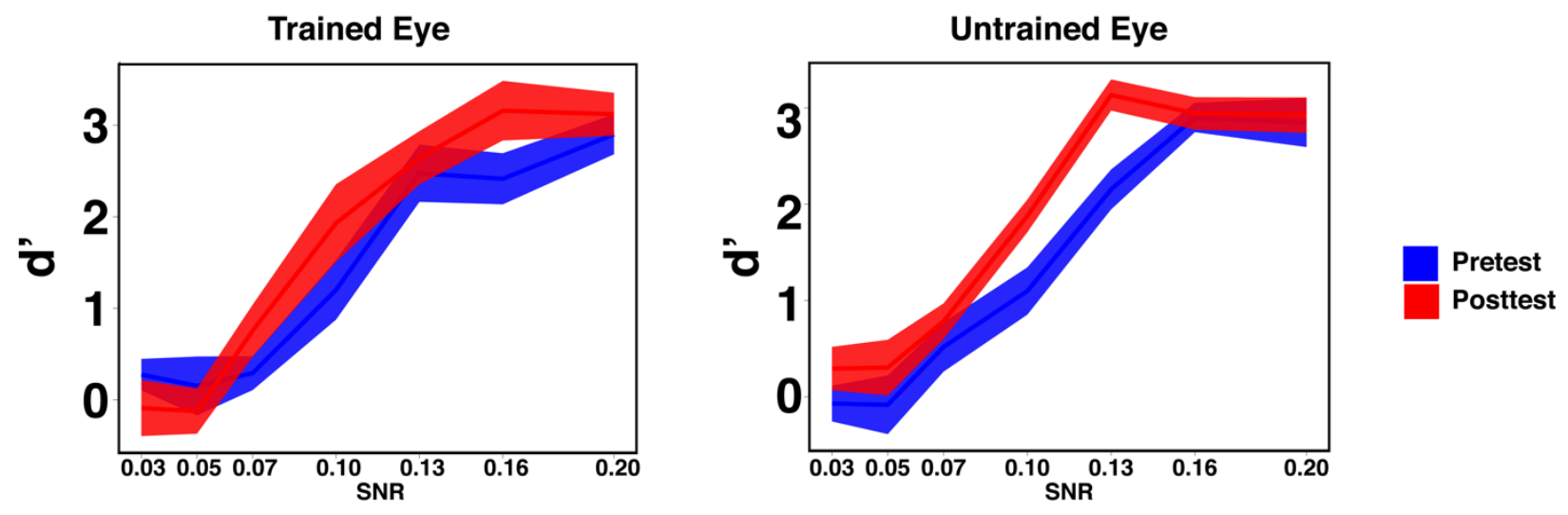

Figure 5. d' of Experiment 2. There was a significant main effect of 'S/N ratio', $F(6,42)=3.012, p<.05$. Post-hoc analysis showed that there was significant improvement in d' in both the trained and untrained eye. 


\section{Discussion}

Our study was aimed to resolve the previous controversy surrounding the role of reward

195 in visual perceptual learning. The reinforcement hypothesis indicates learning should occur only

196 when reward is given subsequently to a stimulus, while according to the alertness hypothesis

197 learning is promoted even when reward precedes the stimulus. In Experiment 1, we conducted

198 two conditions; in the reinforcement condition one of the two orientations that were presented in

199 sequence was presented prior to reward and in the alertness condition it preceded reward. The

200 results demonstrated that VPL of the orientation occurred only when the stimulus preceded

201 reward. This is in accordance with the reinforcement learning hypothesis. Furthermore, VPL was

202 restricted to the trained eye, which suggests that early visual areas are associated with

203 reinforcement-driven VPL. We also conducted the Experiment 2 in which one of the two

204 orientations was presented with a beep sound prior to it. We found improvement in the

205 orientation that was paired with sound which rules out the possibility that alertness before

206 stimulus is not sufficient for performance improvement.

207 Previous evidence demonstrated that reward evokes both reinforcement signals (Wolfram

208 Schultz, 2006) and alertness (Aston-Jones \& Cohen, 2005). Nevertheless, it seems that at least in

209 the current experimental setting VPL is driven by reinforcement processing but not by alertness.

210 In our study, we attempted to increase the possibility of VPL by introducing a 100ms overlap

211 between the reward and stimulus presentation. However, VPL did not occur despite the

212 overlapping interval in the reward before stimulus group. Therefore, the contiguity requirement-

213 reward following the stimulus- seems to be a prerequisite for reward elicited learning to occur.

214 When alertness is directly manipulated through sound, we not only found improvement

215 of the trained orientation in the trained eye, we also observed improvement transferred to the 
216 untrained eye. Alertness might increase along with the changes in attention. The transfer of

217 learning may occur as a result of involvement of higher-order processing in the brain as a result

218 of increase in alertness.

219 Although decision areas are typically associated with reinforcement learning, our results

220 that VPL did not transfer to the untrained eye suggests the involvement of early visual areas in

221 reinforcement driven VPL. This is consistent with studies in which reward/ reinforcement

222 processing changes activity in the primary visual cortex. It has been found that reward induces

223 reduced signal in the early visual cortex when reward is predictive of a visual cue (Arsenault et

224 al., 2013). Neurons in the rodents' primary visual cortex learned to be activated to the stimulus

225 that predict reward (Shuler \& Bear, 2006). In addition to the findings with animals, the

226 improvement in d' in our results indicates that the it is not induced by a change in response bias

227 but rather a sensitivity enhancement to the trained features. All of these results suggest that

228 reinforcement processing reaches early visual areas which involved VPL.

229 A number of studies have indicated that reward plays a crucial role in VPL. However, it

230 remained unclear whether reinforcement processing or alertness caused by reward is a major

231 factor on VPL. We conducted experiments in which the temporal order of the presentations of a

232 visual stimulus and reward was varied. We found that VPL of the stimulus occurs when the

233 visual stimulus was presented before reward but not when it followed reward. In addition, such

234 reward-driven VPL did not transfer to the untrained eye. These results suggest that it is

235 reinforcement processing and not alertness which interacts with visual stimulus signals in early

236 visual areas and leads to VPL. 
Method

Participants

241 A total of 26 participants were recruited in this study. 18 participants (13 females) were recruited

242 for Experiment 1 and 8 (6 females) participants were recruited for Experiment 2. Participants

243 were adults (aged between 18 and 60) with normal or corrected-to-normal vision. This study was

244 approved by the Institutional Review Board (IRB) of Brown University. Written consent forms

245 were provided to participants in accordance with the IRB. Participants had never participated in

246 visual training experiments prior to this study.

\section{$248 \quad$ Stimuli}

249 Noise masked sinusoidal gratings were presented during test and training sessions. The gratings

250 were orientated $112.5^{\circ}$ or $22.5^{\circ}$, with $4^{\circ}$ diameter, $10 \%$ contrast and a spatial frequency of 2

251 cycle/degree. The gratings were presented at the center of an $8^{\circ}$ annulus surrounded by gray

252 background. The $4^{\circ}$ to $8^{\circ}$ field of the annulus was presented with Gaussian masked random noise.

253 The stimuli were presented at the center $0^{\circ}$ to $4^{\circ}$ masked by noise. The noise was generated from

254 a sinusoidal luminance distribution, which ensured that the statistical distributions of the

255 luminance for the noise and the gratings were identical to each other. Consequently, there were

256 no texture cues associated with different levels of noises. During test sessions, 7 different signal-

257 to-noise levels (SN: 0.03, 0.05, 0.07, 0.1, 0.13, 0.16 and 0.2$)$ were varied from trial to trial.

258 During training sessions, a constant SN level of 0.2 were used. 
262 Sensitivity tests were performed during Pretest and Posttest sessions. These test sessions were

263 scheduled at least one day apart from the training sessions. During the test sessions, stimuli was

264 presented to one eye and gray screen was presented with the other eye. Participants were

265 instructed to align the two screens before starting each testing block. At the beginning of each

266 trial, random noise was presented with a green fixation point for 500ms. The noise was followed

267 by the grating stimuli and a red fixation point for $500 \mathrm{~ms}$. The red fixation point will be presented

268 for another $2500 \mathrm{~ms}$ after the stimuli disappeared. The red fixation point indicated the presence of

269 stimuli as well as the signal for participants to make responses. Participants were instructed to

270 judge whether $112.5^{\circ}$ or $22.5^{\circ}$ grating was presented by pressing the corresponding buttons. $7 \mathrm{SN}$

271 levels was presented for each of the two orientations in two eyes separately. The 28 conditions

272 were pseudorandomly interleaved with 32 trials for each condition. Participants were tested for a

273 total of 896 trials in total during the pretest and posttest sessions.

275 Training

276 There were twelve training sessions. Each training session took place on different days.

277 Participants were asked to abstain from eating and drinking for five hours before each training

278 session. Water was provided for reward to the participants during the training period.

279 Participants were separated into two groups --Reward Before Stimulus Group and Reward After

280 Stimulus Group - differed in the timing between the delivery of reward and the presentation of

281 grating stimuli. Continuous Flash Suppression (CFS) paradigm (Tsuchiya \& Koch, 2005) was

282 adopted during the training period to render the stimulus invisible to the participants. This

283 procedure eliminated subject's conscious bias of associating grating stimuli with the presence or

284 absence of reward. To ensure contiguous suppression from flash patterns, we adopted an 
285 alternating mini-block of $15 \mathrm{~s}$ CFS stimuli to each eye. During the first mini-block, the untrained

286 eye was presented with high contrast flashing noise. The CFS stimuli consisted of a sequence of

287 full-screen textured pattern images presenting at a rate of $10 \mathrm{~Hz}$. The texture pattern consisted of

288300 randomly placed, physically overlapping rectangles or ellipses of various sizes with

289 dimension from $0.5^{\circ}$ to $5^{\circ}$. The shapes were of different orientations as well as saturated colors $(0$

290 or $100 \mathrm{~cd} / \mathrm{m}^{2}$ ). In addition, $50 \%$ of the screen was covered with spatially sparse, colored noise.

291 The trained eye was presented with $2 \mathrm{~Hz}$ noise. Intermittently, a grating stimulus $\left(112.5^{\circ}\right.$ or $\left.22.5^{\circ}\right)$

292 of $0.2 \mathrm{SN}$ was presented to the trained eye for $500 \mathrm{~ms}$. One of the orientations was chosen

293 randomly as the trained orientation. The time interval between the grating stimulus was at least

$2943000 \mathrm{~ms}$. For the Reward Before Stimulus Group, water was delivered 400ms prior to the

295 presentation of the trained grating stimulus. For the Reward After Stimulus Group, water was

296 presented 400ms following the presentation of trained grating stimulus. No reward was paired

297 with the untrained grating stimulus. In both cases, reward was presented for 500ms with $100 \mathrm{~ms}$

298 overlap with the stimulus presentation. The overlap ensures processing of reward and maximizes

299 the possibility that water reward could be associated with orientation processing. After the first

300 15s mini-block, the trained eye was presented with the CFS stimuli while the untrained eye was

301 presented with a gray screen. No reward was presented during this mini-block. The 15 s mini-

302 blocks alternated for 5 minutes, and participants were asked to take a 3-minute break after the 5-

303 minute block. . These sequences were repeated for 6 times per session, yielding a total of 120

304 trials for the trained and untrained orientations respectively. Participants were requested to adjust

305 the haploscope to align the two screens after each break. 
Awareness Test

309 Awareness test was conducted immediately after the Posttest to ensure the CFS paradigm has

310 successfully rendered the stimuli invisible. Participants were presented with the same stimuli as

311 in training sessions for one block. During the awareness test, participants were asked to decide

312 whether there was a grating presented and the orientation of the grating by pressing

313 corresponding buttons. Participants were also interviewed whether they were able to detect the

314 grating during the awareness test session as well as during the previous training sessions.

315 Participants were also inquired about whether they were aware of any patterns in the timing of

316 water delivery.

\section{Experiment 2}

319 Pretest and Posttest

320 Participants performed the same sensitivity tests during pre- and post-test as in Experiment 1.

\section{Training}

322 Participants were trained for 12 sessions. The procedure was the same as the Reward Before

323 Stimulus Group in Experiment 1 except that a beep sound was presented 400ms before the

324 stimulus presentation instead of reward. Additionally, participants were not instructed to abstain

325 from eating and drinking before each training session.

326 Awareness Test

327 The procedure for Awareness tests was the same as in Experiment 1. 
330 Stimuli were presented on two 19 CRT screens (1024 x 768 pixels, $120 \mathrm{~Hz})$. The luminance

331 of the screen was gamma corrected. The stimuli were presented through MATLAB (The

332 MathWorks, Natick, MA) and Psychtoolbox-3 (Brainard, 1997; Pelli, 1997; Kleiner et al., 2007)

333 using a Mac OSX computer. Participants viewed the stimuli through a haploscope. The viewing

334 distance was $82 \mathrm{~cm}$. The water delivery equipment was ValveLink $\rightarrow 8.2$ system manufactured by

335 Automate Scientific, Inc.

337 Acknowledgements

338 This study was supported by NIH R01EY019466 and NSF BCS 1539717 to Yuka Sasaki.

340 Author Contributions

341 Z.W., Y.S. and T.W. designed the study. Z.W. and D.K. wrote the experimental codes. Z.W.

342 conducted the experiments and analyzed the data. Z.W., Y.S. and T.W. wrote the manuscript.

\section{Declaration of interests}

345 The authors declare no competing interests. 


\section{References}

Arsenault, John T., Nelissen, K., Jarraya, B., \& Vanduffel, W. (2013). Dopaminergic Reward Signals Selectively Decrease fMRI Activity in Primate Visual Cortex. Neuron, 77(6), 11741186. doi:10.1016/j.neuron.2013.01.008

Aston-Jones, G., \& Cohen, J. D. (2005). AN INTEGRATIVE THEORY OF LOCUS COERULEUSNOREPINEPHRINE FUNCTION: Adaptive Gain and Optimal Performance. Annual Review of Neuroscience, 28(1), 403-450. doi:10.1146/annurev.neuro.28.061604.135709

Dinse, H. R., Ragert, P., Pleger, B., Schwenkreis, P., \& Tegenthoff, M. (2003). Pharmacological Modulation of Perceptual Learning and Associated Cortical Reorganization. Science, 301(5629), 91.

Dosher, B., \& Lu, Z.-L. (2017). Visual Perceptual Learning and Models. Annual Review of Vision Science, 3(1), 343-363. doi:10.1146/annurev-vision-102016-061249

Frankó, E., Seitz, A. R., \& Vogels, R. (2010). Dissociable Neural Effects of Long-term StimulusReward Pairing in Macaque Visual Cortex. Journal of Cognitive Neuroscience, 22(7), 1425-1439. doi:10.1162/jocn.2009.21288

Gilbert, C. D., \& Li, W. Adult visual cortical plasticity. (1097-4199 (Electronic)).

Kim, D., Lokey, S., \& Ling, S. (2017). Elevated arousal levels enhance contrast perception. Journal of Vision, 17(2), 14-14. doi:10.1167/17.2.14

Kim, D., Seitz, A. R., \& Watanabe, T. (2015). Visual perceptual learning by operant conditioning training follows rules of contingency. Visual Cognition, 1-14. doi:10.1080/13506285.2015.1015663

Law, C.-T., \& Gold, J. I. (2008). Neural correlates of perceptual learning in a sensory-motor, but not a sensory, cortical area. Nature Neuroscience, 11, 505-513. doi:10.1038/nn2070

Law, C.-T., \& Gold, J. I. (2009). Reinforcement learning can account for associative and perceptual learning on a visual-decision task. Nature Neuroscience, 12, 655-663. doi:10.1038/nn.2304

Levi, D. M. (2012). Prentice Award Lecture 2011: Removing the Brakes on Plasticity in the Amblyopic Brain. Optometry and Vision Science, 89(6), 827-838. doi:10.1097/OPX.0b013e318257a187

Li, W. (2016). Perceptual Learning: Use-Dependent Cortical Plasticity. Annual Review of Vision Science, 2(1), 109-130. doi:10.1146/annurev-vision-111815-114351

Li, W., Piëch, V., \& Gilbert, C. D. (2004). Perceptual learning and top-down influences in primary visual cortex. Nature Neuroscience, 7(6), 651-657. doi:10.1038/nn1255

Posner, M. I., \& Petersen, S. E. The attention system of the human brain. (0147-006X (Print)).

Rescorla, R. A., \& Wagner, A. R. 3 A Theory of Pavlovian Conditioning: Variations in the Effectiveness of Reinforcement and Nonreinforcement. 18.

Rescorla, R. A., \& Wagner, A. R. (1972). A theory of Pavlovian conditioning: Variations in the effectiveness of reinforcement and nonreinforcement. Classical Conditioning II Current Research and Theory, 21(6), 64-99. doi:10.1101/gr.110528.110

Sagi, D. (2011). Perceptual learning in Vision Research. Vision Research, 51(13), 1552-1566. doi:https://doi.org/10.1016/j.visres.2010.10.019

Schultz, W. (2002). Getting formal with dopamine and reward. Neuron, 36, 241-263. doi:10.1016/S0896-6273(02)00967-4 
Schultz, W. (2006). Behavioral Theories and the Neurophysiology of Reward. Annual review of psychology, 57, 87-115. doi:10.1146/annurev.psych.56.091103.070229

Schultz, W. (2007). Behavioral dopamine signals. Trends in Neurosciences, 30(5), 203-210. doi:10.1016/j.tins.2007.03.007

Schultz, W., Dayan, P., \& Montague, P. R. (1997). A Neural Substrate of Prediction and Reward. Science, 275(5306), 1593-1599. doi:10.1126/science.275.5306.1593

Seitz, A. R., \& Dinse, H. R. (2007). A common framework for perceptual learning. Current Opinion in Neurobiology, 17(2), 148-153. doi:https://doi.org/10.1016/j.conb.2007.02.004

Seitz, A. R., Kim, D., \& Watanabe, T. (2009). Rewards Evoke Learning of Unconsciously Processed Visual Stimuli in Adult Humans. Neuron, 61, 700-707. doi:10.1016/j.neuron.2009.01.016

Shuler, M. G., \& Bear, M. F. (2006). Reward timing in the primary visual cortex. Science, 311(5767), 1606-1609. doi:10.1126/science.1123513

Tsuchiya, N., \& Koch, C. (2004). Continuous flash suppression reduces negative afterimages. Nature Neuroscience(1097-6256 (Print)).

Watanabe, T., \& Sasaki, Y. (2015). Perceptual Learning: Toward a Comprehensive Theory. Annual review of psychology(August), 1-25. doi:10.1146/annurev-psych-010814-015214

Xue, X., Zhou, X., \& Li, S. (2015). Unconscious reward facilitates motion perceptual learning. Visual Cognition, 23(1-2), 161-178. doi:10.1080/13506285.2014.981625

Yang, E., Brascamp, J., Kang, M.-S., \& Blake, R. (2014). On the use of continuous flash suppression for the study of visual processing outside of awareness. Frontiers in Psychology, 5(724). doi:10.3389/fpsyg.2014.00724 